\title{
Interacciones temporales y espaciales de mesocarnívoros simpátricos en una Reserva de la Biosfera: ¿coexistencia o competencia?
}

\author{
Rogelio Carrera-Treviño ${ }^{1}$, Claudia C. Astudillo-Sánchez ${ }^{2}$, Héctor A. Garza-Torres ${ }^{3}$, \\ Luis Martínez-García ${ }^{1,3}$ \& Leroy Soria-Díaz ${ }^{3 *}$ \\ 1. Laboratorio de Fauna Silvestre, Facultad de Medicina Veterinaria y Zootecnia, Universidad Autónoma de Nuevo \\ León, Campus Ciencias Agropecuarias, Francisco Villa s/n, Col. Ex-Hacienda "El Canadá", Escobedo, Nuevo León, \\ México, C.P. 66050; rogeliocarrera@hotmail.com, martinez.garcialuisfer@gmail.com \\ 2. Facultad de Ingeniería y Ciencias, Centro Universitario Victoria, Universidad Autónoma de Tamaulipas. Adolfo López \\ Mateos, Ciudad Victoria, Tamaulipas, México, C. P. 87149; ccastudillo@docentes.uat.edu.mx \\ 3. Instituto de Ecología Aplicada, Universidad Autónoma de Tamaulipas, Av. División del Golfo No. 356. Col. Libertad, \\ Ciudad Victoria, Tamaulipas, México, C.P. 87019; hagarza@docentes.uat.edu.mx, leroysoriadiaz@gmail.com \\ * Correspondencia
}

\section{Recibido 02-II-2018. C Corregido 02-V-2018. Aceptado 08-VI-2018.}

\begin{abstract}
Temporal and spatial interactions of sympatric mesocarnivores at a Biosphere Reserve: coexistence or competition? Interspecific interactions among tropical mesocarnivorous species and other mammalian trophic guilds have been poorly studied, despite they have important implications in the survival, structure, demography, and distribution of these species. In this study we analyzed spatio-temporal interactions of three sympatric mesocarnivores that are located in the Northeastern limit of their geographic distribution in Mexico, with the objective of analyzing if these species coexist or compete in the axis of the temporal and spatial niche. With a sampling period from January 2015 to December 2016 and 26 camera-trapping stations (with in a set of two camera traps opposite to each other) and located along roads and animal trails, we determined the activity pattern and habitat use of L. wiedii (margay), L. pardalis (ocelote) y P. yagouaroundi (yaguarundi). All independent photographs of each species were grouped into three temporal categories (day, night and twilight) and six habitat categories; Oak Forest, Oak-Pine Forest, Cloud Forest, Pine-Oak Forest, Tropical Deciduous Forest and Medium Forest. Temporal and spatial overlap between species (i.e. interactions) was obtained with the Czekanowski index and the Pianka index, respectively. These indices are symmetrical and take values from zero to one, where the results close to zero indicate that there is no overlap between species and values close to one indicate overlap. We obtained a total of 379 independent photographs, of which 239 corresponded to margay, 118 to ocelot and 22 to yaguarundi. Margay and ocelot were nocturnal, with $75 \%$ of their records in this category showing a high temporal overlap (0.85); whereas yaguarundi was fully diurnal, suggesting it may be able to coexist with the other two species (margay and ocelot). Moreover, the yaguarundi used habitat similar to ocelot and margay (with high spatial overlap of 0.81 and 0.72 , respectively), while the spatial overlap between the margay and ocelot was intermediate (0.53), since they used in most cases different habitat types. Our results suggest that there is no interspecific competition among these tropical mesocarnivorous species, probably due to antagonistic interactions among them on the temporal and spatial axis. These strategies may positively favor populations of mesocarnivores, which are characterized by having a high territorial behavior. Rev. Biol. Trop. 66(3): 996-1008. Epub 2018 September 01.
\end{abstract}

Key words: "El Cielo" Biosphere Reserve; Tamaulipas; Mexico; tropical felines; interespecific interactions.

Las interacciones interespecíficas entre los carnívoros y otros gremios tróficos de mamíferos, han sido un tema central en la ecología de comunidades, debido a que tienen implicaciones importantes en la supervivencia, estructura, demografía y distribución de las especies (Thompson, 1988; Holt \& Polis, 1997; Atwood, Gese, \& Kunkel, 2007). Estas interacciones tienen dependencia ambiental multifactorial, así como de la abundancia de las 
especies de interés, las presas disponibles, sus competidores, temporadas anuales (lluvia, sequía) y disturbios antrópicos o naturales, entre otros (Thompson, 1988; Durant, 1998; Gómez-Ortiz, Monroy-Vilchis, \& MendozaMartínez, 2015). Un enfoque que con frecuencia se utiliza para analizar las interacciones entre especies que comparten los recursos (alimenticios, de espacio, entre otros), ha sido a través del solapamiento espacio-temporal o alimenticio de su nicho ecológico (Pianka, 1973), donde la teoría predice que un solapamiento alto ya sea espacio-temporal o alimenticio, promueve la competencia entre especies, mientras que un solapamiento bajo en alguno de estos aspectos del nicho ecológico, permite su coexistencia (Gause, 1932; Hardin, 1960).

Las interacciones entre especies han sido analizadas y debatidas por décadas (Macarthur \& Levins, 1967; Schoener, 1974). Sin embargo, en el caso de los mamíferos carnívoros particularmente de México hasta Sudamérica, la mayoría de los estudios se han enfocado en felinos de tallas grandes como jaguares (Panthera onca) y pumas (Puma concolor), de los cuales se han descrito sus interacciones espaciales, temporales y alimentarias (Kortello, Hurd, \& Murray, 2007; Harmsen, Foster, Silver, Ostro, \& Doncaster, 2009; Hass, 2009; Hayward \& Slotow, 2009; Foster et al., 2013), mientras que para las especies de talla mediana o también llamadas mesocarnívoros, como el margay o tigrillo (Leopardus wiedii), el ocelote (Leopardus pardalis) y el yaguarundi (Puma yagouaroundi), se tiene poca información (Roemer, Gompper, \& Van Valkenburgh, 2009; Di Bitetti, De Angelo, Di Blanco, \& Paviolo, 2010; Gómez-Ortiz et al., 2015; Bianchi, Olifiers, Gompper, \& Mourão, 2016; Carvajal-Villareal, 2016; Pérez-Irineo \& Santos-Moreno, 2016). Algunos estudios con felinos medianos y otros mesocarnívoros, indican que estas especies reducen su competencia interespecífica al utilizar intervalos de tiempo y espacio diferentes para evitar encuentros entre ellos y coexistir (Di Bitetti, Di Blanco, Pereira, Paviolo, \& Pérez, 2009; Witczuk, Pagacz, Gliwicz, \& Mills, 2015; Carvajal-Villareal,
2016; Massara, Paschoal, Bailey, Doherty, \& Chiarello, 2016).

Los mesocarnívoros como el ocelote, el tigrillo y el yaguarundi destacan entre otras especies como parte esencial de la estructura de las comunidades donde se distribuyen debido a que regulan las poblaciones de herbívoros pequeños, y en ausencia de carnívoros grandes como el jaguar o el puma, pueden llegar a ser los depredadores tope, es decir, pueden llegar a ocupar la cúspide de la cadena trófica (Roemer et al., 2009). Sin embargo, estos mesocarnívoros en particular, también se caracterizan por ser especies crípticas, huidizas y con densidades bajas, lo que ha dificultado su estudio y la generación de información ecológica (Trolle, 2003; Di Bitetti, Paviolo, \& De Angelo, 2006). Afortunadamente, en la actualidad el uso de trampas-cámara ha facilitado el estudio de los mamíferos silvestres tanto a nivel mundial, como en México, donde el uso de esta herramienta ha permitido que el registro y el análisis de la distribución geográfica de los mesocarnívoros en este país vaya en aumento (Botello, Rangel, Linaje, \& Cordero, 2006; Bárcenas \& Medellín, 2010; Ahumada-Carrillo, Monroy, \& Iñiguez, 2013; Almazán-Catalán et al., 2013; Aranda, Botello, Martínez-Meyer, \& Pineda, 2014; Aranda \& Valenzuela-Galván, 2015). Aún así, son pocos los trabajos que han estudiado sus interacciones temporales y espaciales (González, Brown, \& Gallo-Reynoso, 2003; Martínez-Calderas et al., 2011; CarvajalVillarreal, Caso, Downey, Moreno, Tewes, \& Grassman, 2012; Pérez-Irineo \& Santos-Moreno, 2014; Carvajal-Villarreal, 2016; PérezIrineo \& Santos-Moreno, 2016).

La distribución actual de los mesocarnívoros considerados para este estudio indica que el margay se encuentra desde el norte de México hasta Uruguay (Vanderhoff, Hodge, Arbogast, Nilsson, \& Knowles, 2011), la Unión Internacional para la Conservación de la Naturaleza (IUCN) lo considera una especie amenazada con una disminución importante en sus poblaciones (Payan et al., 2008), mientras que en México está en peligro de extinción (Semarnat, 2010). El ocelote se distribuye desde el sur de 
Texas hasta el norte de Argentina (Murray \& Gardner, 1997; Sunquist \& Sunquist, 2002), la IUCN lo clasifica como una especie de preocupación menor y en México está catalogado en peligro de extinción (Semarnat, 2010). El yaguarundi cuenta con la distribución geográfica más amplia entre los mesocarnívoros de este estudio, la cual abarca desde el sur de Estados Unidos hasta la parte central de Argentina (Giordano, 2016), es considerado de preocupación menor para la IUCN (Caso et al., 2008), y una especie amenazada por la legislación ambiental mexicana (Semarnat, 2010).

En México, el estado de Tamaulipas representa uno de los límites Noreste de la distribución geográfica de estos tres mesocarnívoros simpátricos (Sunquist \& Sunquist, 2002; Vargas-Contreras \& Hernández-Huerta, 2001). En este estado se localiza la Reserva de la Biosfera El Cielo (RBC), la cual es una zona importante por albergar gran cantidad de endemismos y diversidad de especies lo que es una muestra del buen estado de conservación que hasta la fecha ha mantenido esta zona de protección biológica (Vargas-Contreras \& Hernández-Huerta, 2001; Steinberg, Taylor, \& Kinney, 2014). Sin embargo, existe poca información sobre los mesocarnívoros que se encuentran en esta Reserva, y hasta el momento sólo se ha analizado la distribución altitudinal de la mastofauna (Vargas-Contreras \& Hernández-Huerta, 2001) y un estudio sobre el patrón de distribución espacial del margay (Carvajal-Villarreal et al., 2012). Esto evidencia un conocimiento somero o nulo acerca de las interacciones interespecíficas (espaciales y temporales), así como de las estrategias de supervivencia que emplean los mesocarnívoros en la RBC. Debido a que la RBC se encuentra en un buen estado de conservación, se planteó la hipótesis que sugiere que no existe competencia espacio-temporal entre los mesocarnívoros de estudio. Por lo tanto, los objetivos de la presente investigación fueron estimar el patrón de actividad y uso de hábitat del margay, ocelote y yaguarundi, para analizar sus interacciones temporales y espaciales a través del análisis de solapamiento de nicho y conocer si estas especies coexisten o compiten en la RBC.

\section{MATERIALES Y MÉTODOS}

Área de estudio: La RBC se localiza en el Noreste de México, en la región biogeográfica de la Sierra Madre Oriental (SMO) sus coordenadas extremas son $22^{\circ} 55^{\prime} \mathrm{N} \& 99^{\circ} 26^{\prime} \mathrm{W}$, y tiene una extensión territorial de $1445 \mathrm{~km}^{2}$ aproximadamente. La RBC es una zona importante de transición entre la región Neártica y Neotropical con una amplitud altitudinal entre 100 y 2300 m (Steinberg et al., 2014), lo que le confiere presentar diferentes condiciones ambientales y tipos de vegetación bien delimitados que se mencionan a continuación con su cobertura vegetal respectiva en la reserva: Bosque de Encino 20 \% (BE); Bosque de Pino Encino 10.55 \% (BPE); Bosque de Encino Pino $7.5 \%$ (BEP); Bosque Mesófilo de Montaña $10.26 \%$ (BMM); Matorral Submontano 10 \% (MS); Mezquital $0.08 \%$ (M); Selva Baja Caducifolia $13.9 \%$ (SBC); Selva Mediana 13.9 \% (SM); Matorral Desértico Rosetófilo 4.34 $\%$ (MDR); Pastizal $7.7 \%$ (P) y Vegetación de Galería $1 \%$ (VG). También presenta áreas de Agricultura $0.67 \%$ (A) (González-Medrano, 2005; INEGI serie V). En la zona de estudio se presenta una estación lluviosa de abril a octubre y una estación de sequía de noviembre a marzo. Por otro lado, también es importante destacar que en la RBC existe la presencia de los seis felinos registrados para México (jaguar, puma, margay, ocelote, yaguarundi y lince).

Muestreo con trampas-cámara: Este estudio se realizó durante dos años de muestreo (enero 2015 a diciembre 2016), se colocaron un total de 26 estaciones dobles de trampeo equipadas con dos trampas-cámara puestas frente a frente y distribuidas en caminos y veredas (Harmsen, Foster, Silver, Ostro, \& Doncaster, 2010) en los diferentes tipos de vegetación de la RBC. Debido a la topografía accidentada y el acceso complicado a los sitios de estudio, las estaciones de trampeo se colocaron en seis tipos de vegetación (BE, BPE, BEP, BMM, 
SBC y SM), con una distancia de separación entre estaciones de 3 a $5 \mathrm{~km}$. Las trampas cámara que se utilizaron fueron de la marca Scoutguard HCO modelo SG565, con sensor de movimiento y de temperatura, éstas se sujetaron a troncos de árboles a 30 o $50 \mathrm{~cm}$ del suelo y se programaron para que funcionaran las 24 horas del día e imprimieran la hora y la fecha de la fotografía capturada. Las trampascámara se revisaron mensualmente.

Análisis de fotografías: Las fotografías se clasificaron como eventos independientes de la forma siguiente: 1) fotografías consecutivas de individuos diferentes (si los individuos se pudieron distinguir por sus patrones de manchas) y 2) fotografías consecutivas de la misma especie separadas por más de 24 horas este criterio se utilizó cuando no fue fácil identificar, a los individuos (O' Brien, Kinnaird, \& Wibisono, 2003).

Análisis del patrón de actividad: Para realizar los análisis estadísticos del patrón de actividad, las fotografías independientes se agruparon en 24 categorías de una hora, lo que representó un día completo. Para describir la actividad diaria de las especies se consideraron tres categorías: día, noche y crepúsculo que incluyó el amanecer y el atardecer. Estas categorías se clasificaron con el programa Sun Times v 7.1 (Kay \& Du Croz, 2008; http:// www.apt172.dsl.pipex.com/suntimes.htm), el cual considera la latitud en la que se encuentra la RBC. Como referencia para establecer los intervalos de las categorías, al horario del amanecer y atardecer se le agregó una hora antes y una hora después (Carbajal-Borges, GodínezGómez, \& Mendoza, 2014). Para saber si el patrón de actividad de cada especie es homogéneo a lo largo de las 24 categorías se utilizó la prueba de Rayleigh (Zar, 2010), en el programa Oriana V4.05, y para saber si había diferencias en el patrón de actividad de cada especie entre los años de muestreo y las estaciones del año, se utilizaron pruebas de Chi-cuadrada (Spiegel, 1991).
El solapamiento temporal de los mesocarnívoros se analizó con la comparación entre pares de especies, a través del índice de Czekanowski (Feisinger, Spears, \& Poole, 1981). Este índice es simétrico y toma valores de cero a uno, donde los resultados cercanos a cero indican que no hay solapamiento entre especies, mientras que los valores cercanos a uno indican solapamiento. El promedio de solapamiento calculado entre los pares de especies de este índice se comparó con el promedio del índice simulado de Czekanowski, el cual se generó con modelos nulos a través de simulaciones con 10000 repeticiones. La comparación entre estos índices (calculado y simulado) indica si el solapamiento en la actividad se debe al azar o no. El índice de Czekanowski y las simulaciones se calcularon con el programa "Time Overlap" con el algoritmo ROSARIO (http:// hydrodictyon.eeb.uconn.edu/people/willig/ Research/activity\%20pattern.html), debido a que este último mantiene la estructura cíclica del tiempo mientras genera los escenarios de simulación y por ello, es adecuado para realizar los análisis de solapamiento temporal (CastroArellano, Lacher, Willig, \& Rangel, 2010).

Análisis del uso de hábitat: Los registros de las fotografías independientes se agruparon en seis tipos de vegetación (BE, BPE, BEP, BMM, SBC y SM). Para saber si existían diferencias en el uso de hábitat de cada especie entre los años de muestreo y las estaciones del año, se utilizaron pruebas de Chi-cuadrada (Spiegel, 1991).

El solapamiento espacial de los mesocarnívoros se analizó entre pares de especies con el índice de Pianka, el cual toma valores de cero a uno $(0=$ no existe solapamiento y $1=$ solapamiento). Estos análisis se calcularon con el programa EcoSim 7.0 (http://www.garyentsminger.com/ecosim/) (Entsminger, 2014). El índice de Pianka (calculado) se comparó con el promedio de este mismo índice (simulado), el cual se obtuvo de los modelos nulos generados con 10000 repeticiones, a través del algoritmo RA3, el cual mantiene la estructura lineal 
cuando genera las simulaciones (Winemiller \& Pianka, 1990).

Finalmente, para observar gráficamente el solapamiento temporal (patrón de actividad) y espacial (tipo de vegetación) de los tres mesocarnívoros, se utilizó un análisis de correspondencia en cada caso. Este análisis permite ordenar la frecuencia de los registros independientes de cada especie en función de las categorías establecidas (día, noche y crepúsculo o BE, BPE, BEP, BMM, SBC, y SM).

\section{RESULTADOS}

Con un esfuerzo total de muestreo de 16023 días-trampa, se lograron obtener 379 fotografías independientes de los tres mesocarnívoros, de las cuales 239 correspondieron a $L$. wiedii, 118 a L. pardalis y sólo 22 a P. yagouaroundi. Las tres especies presentaron actividad heterogénea a lo largo del día ( $L$. wiedii, $\mathrm{Z}=$ $10.88, \mathrm{P}<0.01 ;$ L. pardalis, $\mathrm{Z}=9.85, \mathrm{P}<0.01$; P. yagouaroundi, $\mathrm{Z}=8.65, \mathrm{P}<0.01)$. Las dos primeras especies (L. wiedii y L. pardalis) presentaron un patrón de actividad nocturno con más del $80 \%$ y $75 \%$ de los registros en esta parte del día, respectivamente. El L. pardalis además presentó una tendencia a usar horarios crepusculares $(20 \%)$. Mientras que el $P$. yagouaroundi fue una especie diurna con el 100 $\%$ de sus registros en esta categoría (Fig. 1A y Fig. 1B). Los picos de actividad en L. wiedii se presentaron en el intervalo de las 04:00 a 04:59 y de las 23:00 a las 00:00 h, en L. pardalis de las 00:00 a 00:59 y de las 22:00 a 22:59 h, mientras que en $P$. yagouaroundi fueron de las 09:00 a 09:59 h y 16:00 a 16:59 h (Fig. 1A).

Los resultados de la prueba Chi-cuadrada muestran que no existen diferencias significativas al comparar el patrón de actividad de cada una de las especies de mesocarnívoros entre los diferentes años de muestreo y por estación lluviosa o sequía (Cuadro 1). Para el caso de $P$. yagouaroundi, no fue posible realizar estas comparaciones debido al número bajo de registros $(\mathrm{n}=22)$, que sólo representa alrededor del $6 \%$ del total de las fotografías seleccionadas como registros independientes.

El promedio de solapamiento temporal entre de las tres especies fue bajo (0.35), y significativamente mayor que el simulado $(0.30$, $\mathrm{P}=0.02$ ), lo que indica que los resultados no son aleatorios. La comparación entre pares de especies sugiere que el margay y el ocelote tienen un solapamiento temporal nocturno alto (0.85), mientras que el solapamiento entre margay-yaguarundi (0.06) y ocelote-yaguarundi (0.05) fue bajo.
A

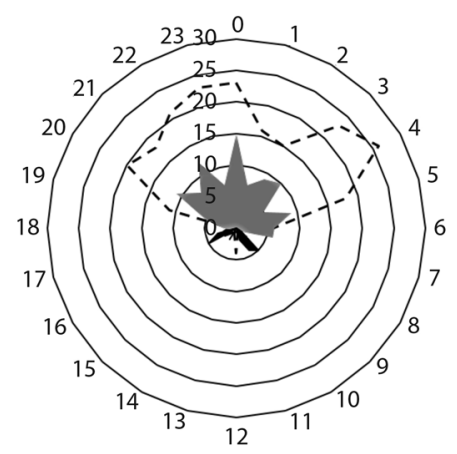

r. L. wiedii

L. pardalis

P. yogouaroundi

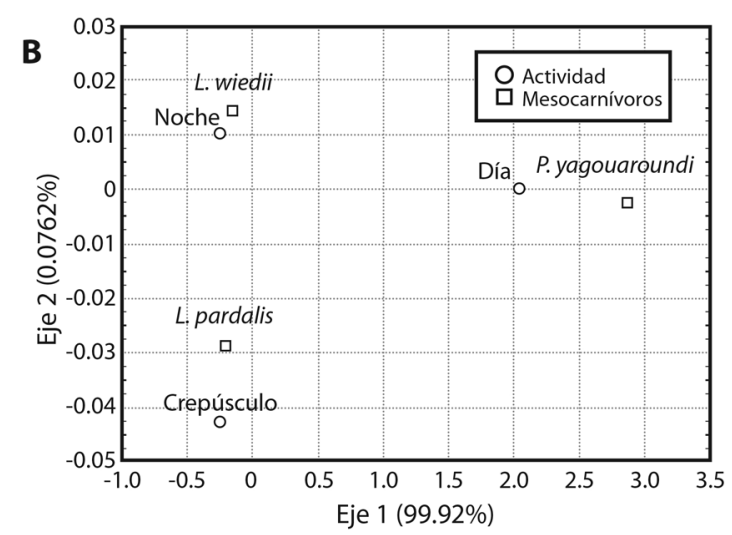

Fig. 1. Patrón de actividad de tres mesocarnívoros presentes en la Reserva de la Biosfera El Cielo, Tamaulipas. A) frecuencia de registros independientes de cada especie en las 24 categorías de horario ( 0 a 23 h). B) análisis de correspondencia de la actividad de cada especie durante el día, la noche y el crepúsculo.

Fig. 1. Activity pattern of mesocarnivores of El Cielo Biosphere Reserve, Tamaulipas. A) frequency of independent records of each species in the 24 time categories ( 0 to $23 \mathrm{~h})$. B) correspondence analysis of the activity of each species during the day, night and twilight. 
CUADRO 1

Comparación interespecífica con análisis de Chi-cuadrada, del patrón de actividad y uso de hábitat de L. wiedii y L. pardalis, en los diferentes años de muestreo (2015-2016) y estación (lluviosa y sequía)

TABLE 1

Interspecific comparison using chi-square analysis of the activity pattern and habitat use of $L$. wiedii and L. pardalis in the different years of sampling (2015-2016) and season (rain-drought)

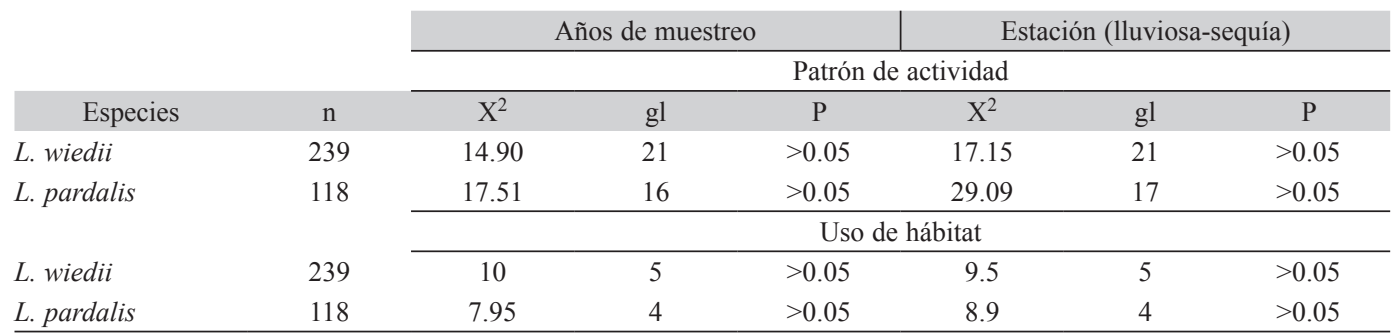

El patrón de actividad de los tres mesocarnívoros es consistente con el análisis de correspondencia, donde se observa que el margay y el ocelote son nocturnos y ambos se separan del yaguarundi el cual es una especie diurna (el eje 1 explica el $99.92 \%$ de la variación de los datos) con una tendencia importante del ocelote a utilizar horarios crepusculares (el eje 2 explica el $0.07 \%$ de la variación de los datos; Fig. 1B).

En cuanto al uso de hábitat, $L$. wiedii registró su actividad principalmente en el BE, L. pardalis en la SBC, y P. yagouaroundi en el $\mathrm{BE}$, con el 34, 54 y $36 \%$ de fotografías independientes, respectivamente (Fig. 2A). No se presentaron diferencias significativas al comparar el uso de hábitat por parte de $L$. wiedii y $L$. pardalis entre los diferentes años de muestreo ni por la estación lluviosa o sequía (Cuadro 1).

Los resultados del índice de Pianka para los tres mesocarnívoros, indica un solapamiento intermedio en el uso de los tipos de vegetación (0.54); no obstante, al comparar con los modelos nulos, se muestra que este valor es significativamente mayor que el promedio de solapamiento simulado $(0.46, \mathrm{P}=0.02)$, esto sugiere que los resultados no son aleatorios. Los valores más altos de solapamiento del uso de hábitat entre pares de especies se encontraron entre L. pardalis y P. yagouaroundi (0.81), seguido de $L$. wiedii y $P$. yagouaroundi $(0.72)$ y los valores más bajos se encontraron entre
L. pardalis y L. wiedii (0.53). El análisis de correspondencia muestra una separación en el uso de hábitat entre L. pardalis y L. wiedii (el eje 1 explicó cerca del $66.73 \%$ de la variación de los datos), mientras que el $P$. yagouaroundi se comporta como una especie generalista al utilizar cuatro tipos de vegetación semejantes a las otras dos especies y probablemente no hay selección por alguno en particular (el eje 2 explicó el $33.27 \%$ de la variación de los datos) (Fig. 2B).

\section{DISCUSIÓN}

Los estudios sobre la actividad temporal de $L$. wiedii son limitados, debido a que no cuentan con un número suficiente de fotografías (menos de 78 fotografías) (Pérez-Irineo \& Santos-Moreno, 2016). En este estudio se lograron obtener 239 fotografías independientes y los resultados del patrón de actividad muestran que $L$. wiedii en la $\mathrm{RBC}$ es una especie nocturna con más del $80 \%$ de sus registros en esta categoría (Fig. 1A), lo cual coincide con lo descrito por Carvajal-Villarreal et al. (2012) para la RBC derivado de un estudio realizado con telemetría. El patrón de actividad de $L$. wiedii es consistente también con regiones tropicales de México como Los Chimalapas Oaxaca (Pérez-Irineo \& Santos-Moreno, 2016), y con zonas de latitudes más bajas como los Andes en Ecuador (Vanderhoff et al., 2011). 


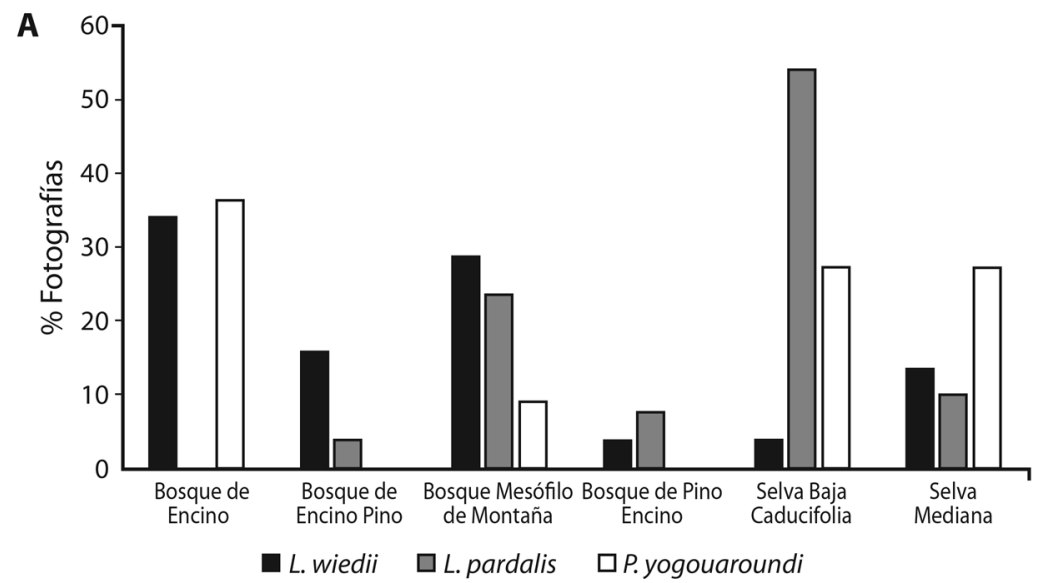

B

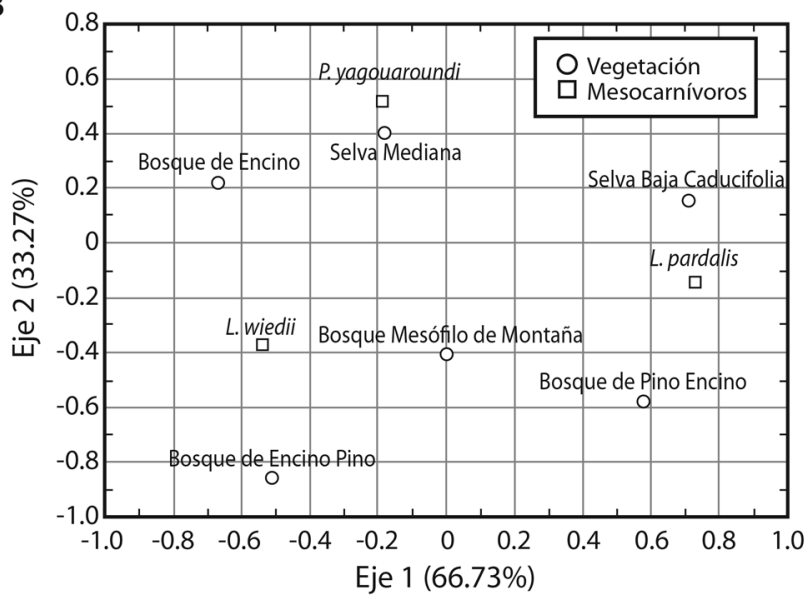

Fig. 2. Uso de hábitat de los mesocarnívoros presentes en la Reserva de la Biosfera El Cielo, Tamaulipas. A) porcentaje de fotografías de cada especie por tipo de vegetación. B) análisis de correspondencia del uso de hábitat.

Fig. 2. Habitat use of mesocarnivores in the El Cielo Biosphere Reserve, Tamaulipas. A) porcentaje of photographs of each species by vegetation type. B) Correspondence analysis of the habitat use.

La actividad nocturna de L. pardalis de este estudio es similar a lo encontrado en otros trabajos de México y Sudamérica (Maffei, Noss, Cuéllar, \& Rumiz, 2005; Di Bitetti et al., 2006; Kolowski \& Alonso, 2010; Carvajal-Villareal, 2016; Porfirio, Foster, Fonseca, \& Sarmento, 2016). Sin embargo, este patrón nocturno del $L$. pardalis es diferente de lo registrado en Los Chimalapas Oaxaca (Pérez-Irineo \& Santos-Moreno, 2016) y en Quintana Roo, México (Torres-Romero Espinoza-Medinilla, Lazcano-Barreto, \& Maffeí, 2017). Estas diferencias pueden explicarse probablemente por la disponibilidad de presas (Pérez-Irineo \& Santos-Moreno, 2016) o por las actividades humanas, que son diferentes en cada sitio de estudio y en la RBC por ejemplo, se considera que hay una baja actividad humana en los sitios donde se realizó el estudio. Finalmente, el patrón de actividad del yaguarundi en la RBC es similar al otros estudios que han determinado que este felino es una especie diurna en la mayor parte de su actividad diaria (Giordano, 2016).

Los resultados del análisis de solapamiento temporal mostraron que $L$. wiedii y $L$. pardalis no se solapan con la actividad del 
P. yagouaroundi (Fig. 1B). Este comportamiento refleja la importancia que tiene la segregación de la actividad temporal como un mediador de las interacciones interespecíficas en estas especies, lo que implica que en la RBC estas especies probablemente no compiten, sino que coexisten en el espacio temporal dentro del ecosistema, lo que favorece mantener poblaciones estables entre estas especies que tienen potencial alto de competir. En estos resultados se destacó en particular la segregación temporal del $P$. yagouaroundi, comportamiento que es semejante con lo observado en México en la Sierra de Tamaulipas y en Los Chimalapas Oaxaca o con la Provincia de Misiones Argentina, donde este felino también utiliza horarios diurnos cuando es simpátrico con otras especies (Di Bitetti et al., 2010; Carvajal-Villareal, 2016; Pérez-Irineo \& Santos-Moreno, 2016).

El solapamiento alto entre la actividad nocturna del margay y el ocelote (0.83), puede deberse a que el ocelote no tiene ningún efecto negativo sobre el margay, ya que se obtuvo un número mayor de fotografías independientes de este último felino (239), lo que indica que en la RBC este felino puede ser más abundante que el ocelote (118 fotografías independientes) y por ello, el margay probablemente no modifica su actividad. Al respecto, De Oliveira et al. (2010), Vanderhoff et al. (2011), Carvajal-Villareal et al. (2012) y Pérez-Irineo \& Santos-Moreno (2016) han argumentado que cuando el margay se encuentra en abundancias mayores que el ocelote, este último no genera ningún efecto sobre las poblaciones de margay y por ello su comportamiento no se ve afectado. Aunado a lo anterior, es posible también que la tendencia que tiene el ocelote a utilizar horarios crepusculares (Fig. 1B) favorece su tolerancia con respecto al margay y por lo tanto pueden coexistir ambos mesocarnívoros en la RBC.

La estacionalidad lluviosa y sequía ha sido considerada como un factor importante que puede modificar la actividad de las especies, debido a la disponibilidad de alimento principalmente (Porfirio et al., 2016), sin embargo, en los resultados de este estudio no hubo evidencia significativa de que las estaciones tuvieran efecto sobre la actividad temporal de L. wiedii y L. pardalis (Cuadro 1). Otros trabajos realizados en latitudes tropicales más bajas del continente americano han encontrado este mismo patrón con el margay en Ecuador (Vanderhoff et al., 2011) y con el ocelote en Brasil (Porfirio et al., 2016), Bolivia (Gómez, Wallace, Ayala, \& Tejada, 2005; Maffei, Cuéllar, \& Noss, 2002), Argentina (Di Bitetti et al., 2006) y Perú (Kolowski \& Alonso, 2010). Tampoco los muestreos en años diferentes tuvieron un efecto sobre la actividad de $L$. wiedii y L. pardalis. Durante los años en los que se realizó el estudio no se registraron cambios o eventos que pudieran alterar el ecosistema de la RBC. Debido a lo mencionado anteriormente se puede inferir que la temporada de lluvia y la sequía y los diferentes años de muestreo (2015-2016), no influyeron en el análisis de solapamiento temporal.

En cuanto al uso de hábitat, los resultados muestran que los valores de solapamiento del índice de Pianka más altos entre pares de especies están entre $L$. pardalis y $P$. yagouaroundi (0.81), seguido de $L$. wiedii y $P$. yagouaroundi (0.72) y los más bajos se encontraron entre $L$. pardalis y L. wiedii (0.53). Estos resultados indican que el yaguarundi se solapa en el eje del nicho espacial con los otros dos mesocarnívoros (Fig. 2B), se esperaría una competencia potencial por el uso del espacio. Sin embargo, en este caso la actividad temporal diurna del yaguarundi le puede permitir utilizar el mismo espacio que las otras dos especies sin que exista una competencia y con ello se puede argumentar que mientras exista un solapamiento bajo en alguno de los ejes del nicho ecológico (temporal, espacial o alimenticio), se puede presentar una coexistencia (Gause, 1932; Hardin, 1960). Este mismo mecanismo de segregación en alguno de los ejes del nicho (temporal o espacial), se ha evidenciado en estudios diversos en donde el yaguarundi es simpátrico con otros felinos (Di Bitetti et al., 2010; Giordano, 2016; Pérez-Irineo \& Santos-Moreno, 2016).

Por otro lado, el margay utilizó el BE y BMM, mientras que el ocelote la $\mathrm{SBC}$ en mayores porcentajes (Fig. 2A) y mostraron 
un índice de Pianka de solapamiento espacial intermedio (0.53) entre las dos especies. Estas preferencias de vegetación de cada especie sugieren que existe una segregación por el uso de hábitat entre ellas (Fig. 2B), lo que destaca la importancia de este recurso como un mediador de las interacciones interespecíficas espaciales. En este caso en particular, aunque en el nicho temporal pudieran solaparse entre margay y ocelote (0.83) en el espacial (uso de hábitat) no lo hacen y por tanto se argumenta la idea de que hay una coexistencia entre estas especies ya que por lo menos hay un solapamiento bajo en alguno de los ejes del nicho ecológico (Gause, 1932; Hardin, 1960).

Las tres especies de mesocarnívoros analizadas en este estudio, han sido asociadas a una gran cantidad de tipos de vegetación conservados, con afinidad a los bosques tropicales y subtropicales, tanto caducifolios como perennifolios; aunque también han sido asociados en algunos casos a zonas abiertas con perturbación humana alta (De Oliveira, 1998; Sunquist \& Sunquist, 2002; Martínez-Calderas et al., 2011; Martínez-Calderas, Rosas-Rosas, Palacio-Núñez, Martínez-Montoya, \& VillordoGalván, 2012; Aranda \& Valenzuela-Galván, 2015; Giordano, 2016). En la Reserva de la Biosfera El Cielo, el margay estuvo asociado a tipos de vegetación conservados (BE y BMM) (Carvajal-Villareal et al., 2012), mientras que el ocelote y el yaguarundi se asociaron a tipos de vegetación con perturbación humana ligera como la SBC y la SM (Fig. 2A). La cercanía que tienen estos últimos tipos de vegetación a los asentamientos humanos pueden estar afectando a los mesocarnívoros de este estudio, sin embargo en nuestros resultados no es evidente el efecto humano.

Durante el periodo de muestreo de este estudio, en la SBC y SM, se registraron ataques de los tres mesocarnívoros involucrados en este estudio, hacia las aves de corral, debido a que se carece de un resguardo adecuado para estos animales domésticos. Por ello, es necesario implementar programas que ayuden a concientizar a los pobladores sobre la importancia que tienen los mesocarnívoros en el ecosistema y proporcionar información para que se tenga un mejor resguardo de los animales domésticos. Al respecto, se están llevando a cabo programas de conservación para disminuir el conflicto entre jaguares y ganaderos de la RBC (Carrera-Treviño, Lira-Torres, MartínezGarcía, \& López-Hernández, 2016) sin embargo, es necesario también tomar medidas de protección para otras especies de carnívoros de tamaño menor como es el caso de los mesocarnívoros involucrados en este estudio.

Aunque en la RBC existen otros tipos de vegetación naturales diferentes a los que se consideraron en este estudio, estos no se lograron incluir en los análisis, debido a que las trampas cámara que se colocaron en estos tipos de vegetación, fueron hurtadas y no se logró obtener evidencia fotográfica. Estos hechos muestran que la presión humana es un aspecto importante a considerar, por lo que resulta necesario aplicar otros métodos y técnicas de monitoreo, que permitan obtener información ecológica sobre las especies de este estudio y el impacto humano que hay sobre la fauna silvestre en la RBC.

Finalmente, los resultados de este estudio muestran que el margay, el ocelote y el yaguarundi coexisten en la RBC. Debido a las interacciones interespecíficas antagonistas que exhiben entre ellos en el tiempo y espacio, es probable que no exista una competencia entre estos mesocarnívoros, lo cual puede favorecer positivamente a las poblaciones de estas especies presentes en la reserva. Sin embargo, es necesario contemplar analizar el eje del nicho trófico entre estas especies para asegurar con mayor precisión las conclusiones que se presentan.

\section{AGRADECIMIENTOS}

Los autores Agradecen al Programa de Conservación de Especies en Riesgo de la Comisión Nacional de Áreas Naturales Protegidas (CONANP), a los propietarios de los terrenos quienes facilitaron el acceso a los mismos, al equipo de trabajo y voluntarios del Laboratorio de Fauna Silvestre de la Facultad 
de Medicina Veterinaria y Zootecnia de la Universidad Autónoma de Nuevo León, quienes ayudaron en la realización de este estudio. Al Consejo Nacional de Ciencia y Tecnología (CONACYT) por la beca posdoctoral otorgada (CVU 255820/LSD), al proyecto financiado por la Universidad Autónoma de Tamaulipas (UAT/PFI2015-15) y al Programa para el Desarrollo Profesional Docente para el Tipo Superior PRODEP (UAT-PTC-221/511-6/17-8212).

\section{RESUMEN}

Las interacciones interespecíficas entre especies de mesocarnívoros tropicales han sido poco estudiadas, a pesar de tener implicaciones importantes en la supervivencia, estructura, demografía, y distribución de estas especies. En este estudio se analizaron las interacciones espacio-temporales de tres mesocarnívoros simpátricos que se encuentran en el límite Noreste de su distribución geográfica en México, con el objetivo de determinar si estas especies coexisten o compiten. Con un periodo de muestreo de enero 2015 a diciembre 2016 y con un total de 26 estaciones de trampeo equipadas con dos trampascámara puestas frente a frente y ubicadas en caminos y veredas dentro de los diferentes tipos de vegetación que presenta la Reserva de la Biosfera El Cielo, se determinó el patrón de actividad y uso de hábitat de L. wiedii (margay), L. pardalis (ocelote) y P. yagouaroundi (yaguarundi). Las fotografías independientes de cada especie se agruparon en tres categorías temporales (día, noche y crepúsculo) y en seis categorías espaciales (tipo de vegetación) para el uso del hábitat; Bosque de Encino, Bosque de Pino Encino, Bosque de Encino Pino, Bosque Mesófilo de Montaña, Selva Baja Caducifolia y Selva Mediana. El solapamiento entre especies (o interacciones) temporales y espaciales se obtuvieron con el índice de Czekanowski y el índice de Pianka, respectivamente. Estos índices son simétricos y toman valores de cero a uno, donde los resultados cercanos a cero indican que no hay solapamiento entre especies y los valores cercanos o con valor de uno indican solapamiento. Se obtuvieron un total de 379 fotografías independientes, de las cuales 239 correspondieron al margay, 118 a ocelote y 22 para el yaguarundi. Se documentó que el margay y el ocelote fueron nocturnos, más del $80 \%$ de sus registros se presentaron en esta categoría y fueron también los que mostraron un solapamiento temporal alto (0.85), en contraste, el yaguarundi fue una especie con actividad totalmente diurna, lo que muestra que probablemente coexiste con las otras dos especies. El yaguarundi utilizó tipos de hábitat similares al ocelote y al margay (con un solapamiento de 0.81 y 0.72 , respectivamente), mientras que el solapamiento espacial entre el margay y el ocelote fue intermedio (0.53), debido a que utilizaron distintos tipos de hábitat en la mayoría de los casos. Los resultados indican que no hay competencia interespecífica entre las especies de mesocarnívoros tropicales de este estudio, esto probablemente se debe a las interacciones antagonistas que exhiben entre ellos ya sea en el eje temporal o espacial. Estas estrategias pueden favorecer positivamente a las poblaciones de estos mesocarnívoros, los cuales se caracterizan por tener un comportamiento territorial alto.

Palabras clave: Reserva de la Biosfera El Cielo; Tamaulipas; México; felinos tropicales; interacciones interespecíficas.

\section{REFERENCIAS}

Ahumada-Carrillo, I. T. A., Monroy, J. C. A., \& Iñiguez, M. A. (2013). Presence of the ocelot (Leopardus pardalis) in northern Jalisco, Mexico. Revista mexicana de biodiversidad, 84, 718-721.

Almazán-Catalán, J. A., Sánchez-Hernández, C., Ruíz-Gutiérrez, F., Romero-Almaraz, M. D. L., Taboada-Salgado, A., Beltrán-Sánchez, E., \& Sánchez-Vázquez, L. (2013). Registros adicionales de felinos del estado de Guerrero, México. Revista mexicana de biodiversidad, 84(1), 347-359. DOI: $10.7550 / \mathrm{rmb} .23087$

Aranda, M., Botello, F., Martínez-Meyer, E., \& Pineda, A. (2014). Primer registro de ocelote (Leopardus pardalis) en el Parque Nacional Lagunas de Zempoala, Estado de México y Morelos, México. Revista Mexicana de Biodiversidad, 85(4), 1300-1302.

Aranda, M. \& Valenzuela-Galván, D. (2015). Registro notable de margay (Leopardus wiedii) en el bosque mesófilo de montaña de Morelos, México. Revista Mexicana de Biodiversidad, 86, 1110-1112.

Atwood, T. C., Gese, E. M., \& Kunkel, K. E. (2007). Comparative Patterns of Predation by Cougars and Recolonizing Wolves in Montana's Madison Range. Journal of Wildlife Management, 71(4), 1098-1106. DOI:10.2193/2006-102

Bárcenas, H., \& Medellín, R. A. (2010). Ocelot (Leopardus pardalis) in Aguascalientes, Mexico. The Southwestern Naturalist, 55(3), 447-449.

Bianchi, R. D. C., Olifiers, N., Gompper, M. E., \& Mourão, G. (2016). Niche Partitioning among Mesocarnivores in a Brazilian Wetland. PLoS ONE, 11(9), e0162893. DOI:10.1371/journal.pone.0162893

Botello, F., Rangel, P. I., Linaje, M., \& Cordero, V. S. (2006). Primer registro del tigrillo (Leopardus wiedii, Schinz 1821) y del gato montés (Lynx rufus, Kerr 1792) en la reserva de la biosfera de Tehuacán-Cuicatlán, Oaxaca, México. Acta Zoológica Mexicana (nueva serie), 22(1), 135-139. 
Carbajal-Borges, J. P, Godínez-Gómez, O., \& Mendoza, E. (2014). Density, abundance and activity patterns of the endangered tapirus bairdii in one of its last strongholds in Southern Mexico. Tropical Conservation Science, 7(1), 100-114. DOI: $10.1177 / 194008291400700102$

Carrera-Treviño, R., Lira-Torres, I., Martínez-García, L., \& López-Hernández, M. (2016). The jaguar Panthera onca (Carnivora: Felidae) in "El Cielo" Biosphere Reserve, Tamaulipas, Mexico. Revista de Biología Tropical, 64(4), 1451-1468. DOI:10.15517/rbt. v64i4.21880

Carvajal-Villarreal, S. (2016). Selected ecological patterns and distribution of five simpatric felid in northeastern Mexico (Doctoral dissertation) Texas A\&M University-Kingsville, USA.

Carvajal-Villarreal, S., Caso, A., Downey, P., Moreno, A., Tewes, M. E., \& Grassman, L. I. (2012). Spatial patterns of the margay (Leopardus wiedii; Felidae, Carnivora) at "El Cielo" Biosphere Reserve, Tamaulipas, Mexico. Mammalia, 76, 237-244.

Caso, A., Lopez-Gonzalez, C., Payan, E., Eizirik, E., De Oliveira, T., Leite-Pitman, R., Kelly, M., \& Valderrama, C. (2008). Herpailurus yagouaroundi. IUCN Red List of Threatened Species, Version 2014.3. Retrieved from www.iucnredlist.org

Castro-Arellano, I., Lacher, T. E., Willig, M. R., \& Rangel, T. F. (2010). Assessment of assemblage-wide temporal niche segregation using null models. Methods in Ecology and Evolution, 1(3), 311-318. DOI: 10.1111/j.2041-210X.2010.00031.x

De Oliveira, T. G. (1998). Margay. Mammalian Species, $579,1-6$.

De Oliveira, T. G., Tortato, M. A., Silveira, L., Kasper C. B., Mazim, F. D., Lucherini M., . . . Sunquist, M. (2010). Ocelot ecology and its effect on the small-felid guild in the lowland neotropic. In D. W. Macdonald \& A. J. Loveridge (Eds.), Biology and conservation of wild felid (pp. 559-596). New York: Oxford University Press.

Di Bitetti, M. S., De Angelo, C. D., Di Blanco, Y. E., \& Paviolo, A. (2010). Niche partitioning and species coexistence in a Neotropical felid assemblage. Acta Oecologica, 36(4), 403-412. DOI: 10.1016/j. actao.2010.04.001

Di Bitetti, M. S., Di Blanco, Y. E., Pereira, J. A., Paviolo, A., \& Pérez, I. J. (2009). Time Partitioning Favors the Coexistence of Sympatric Crab-Eating Foxes (Cerdocyon thous) and Pampas Foxes (Lycalopex gymnocercus). Journal of Mammalogy, 90(2), 479490. DOI: 10.1644/08-MAMM-A-113.1

Di Bitetti, M. S., Paviolo, A., \& De Angelo, C. (2006). Density, habitat use and activity patterns of ocelots (Leopardus pardalis) in the Atlantic Forest of
Misiones, Argentina. Journal of Zoology, 270(1), 153-163. DOI: 10.1111/j.1469-7998.2006.00102.x

Durant, S. M. (1998). Competition refuges and coexistence: an example from Serengeti carnivores. Journal of Animal Ecololy, 67, 370-386.

Entsminger, G. L. (2014). EcoSim Professional: Null modeling software for ecologists, Version 1. Acquired Intelligence Inc., Kesey-Bear, \& Pinyon Publishing. Montrose, CO 81403. Retrieved from http:// www.garyentsminger.com/ecosim/index.htm

Feisinger, P., Spears, E. E., \& Poole, R. W. (1981). A simple measure of niche breadth. Ecology, 62, 27-32.

Foster, V. C., Sarmento, P., Sollmann, R., Tôrres, N., Jácomo, A. T. A., Negrões, N., ... Silveira, L. (2013). Jaguar and Puma Activity Patterns and Predator-Prey Interactions in Four Brazilian Biomes. Biotropica, 45(3), 373-379. DOI: 10.1111/btp.12021

Gause, G. F. (1932). Experimental Studies on the Struggle for Existence. Journal of Experimental Biology, 9(4), 389-402.

Giordano, A. J. (2016). Ecology and status of the jaguarundi Puma yagouaroundi: a synthesis of existing knowledge. Mammal Review, 46(1), 30-43. DOI: 10.1111/mam. 12051

Gómez, H., Wallace, R. B., Ayala, G., \& Tejada, R. (2005). Dry season activity periods of some Amazonian mammals. Studies on Neotropical Fauna and Environment, 40(2), 91-95. DOI: 10.1080/01650520500129638

Gómez-Ortiz, Y., Monroy-Vilchis, O., \& Mendoza-Martínez, G. D. (2015). Feeding interactions in an assemblage of terrestrial carnivores in central Mexico. Zoological Studies, 54(1), 16. DOI: 10.1186/ s40555-014-0102-7

González, C. A. L., Brown, D. E., \& Gallo-Reynoso, J. P. (2003). The ocelot Leopardus pardalis in north-western Mexico: ecology, distribution and conservation status. Oryx, 37(03), 358-364.

González-Medrano, F. (2005). La vegetación. In G. Sánchez-Ramos, P. Reyes-Castillo, \& R. Dirzo (Eds.), Historia Natural de la Reserva de la Biosfera El Cielo, Tamaulipas, México (pp. 88-105). México: Universidad Autónoma de Tamaulipas.

Hardin, G. (1960). The Competitive Exclusion Principle. Science, 131, 1292-1297.

Harmsen, B. J., Foster, R. J., Silver, S., Ostro, L., \& Doncaster, C. P. (2009). Spatial and temporal interactions of sympatric jaguars (Panthera onca) and pumas (Puma concolor) in a neotropical forest. Journal of Mammalogy, 90(3), 612-620.

Harmsen, B. J., Foster, R. J., Silver, S., Ostro, L., \& Doncaster, C. P. (2010). Differential Use of Trails by Forest 
Mammals and the Implications for Camera-Trap Studies: A Case Study from Belize. Biotropica, 42(1), 126-133. DOI: 10.1111/j.1744-7429.2009.00544.x

Hass, C. C. (2009). Competition and coexistence in sympatric bobcats and pumas. Journal of Zoology, 278(3), 174-180. DOI: 10.1111/j.1469-7998.2009.00565.x

Hayward, M. W., \& Slotow, R. (2009). Temporal Partitioning of Activity in Large African Carnivores: Tests of Multiple Hypotheses. South African Journal of Wildlife Research, 39(2), 109-125. DOI: $10.3957 / 056.039 .0207$

Holt, R. D., \& Polis, G. A. (1997). A Theoretical Framework for Intraguild Predation. The American Naturalist, 149(4), 745-764. DOI: 10.1086/286018

INEGI (Instituto Nacional de Estadística y Geografía, serie V), uso de suelo y vegetación escala 1:250 000. Recuperado de http://www.inegi.org.mx/geo/contenidos/ recnat/usosuelo/

Kay, S., \& Du Croz, T. (2008). Sun Times. Version 7.1. Retrieved from http://www.aptl72. dsl.pipex.com/ suntimes.htm

Kolowski, J. M., \& Alonso, A. (2010). Density and activity patterns of ocelots (Leopardus pardalis) in northern Peru and the impact of oil exploration activities. Biological Conservation, 143(4), 917-925. DOI: 10.1016/j.biocon.2009.12.039

Kortello, A. D., Hurd, T. E., \& Murray, D. L. (2007). Interactions between cougars (Puma concolor) and gray wolves (Canis lupus) in Banff National Park, Alberta. Écoscience, 14(2), 214-222. DOI: 10.2980/1195-6860(2007)14[214:IBCPCA]2.0.CO;2

Macarthur, R., \& Levins, R. (1967). The Limiting Similarity, Convergence, and Divergence of Coexisting Species. The American Naturalist, 101(921), 377385. DOI:10.1086/282505

Maffei, L., Cuéllar, E., \& Noss, A. J. (2002). Uso de trampas-cámara para la evaluación de mamíferos en el Ecotono Chaco-Chiquitanía. Revista Boliviana de Ecología y Conservación Ambiental, 11, 55-65.

Maffei, L., Noss, A. J., Cuéllar, E., \& Rumiz, D. I. (2005). Ocelot (Felis pardalis) population densities, activity, and ranging behaviour in the dry forests of eastern Bolivia: data from camera trapping. Journal of Tropical Ecology, 21(03), 349-353. DOI:10.1017/ S0266467405002397

Martínez-Calderas, J. M., Rosas-Rosas, O. C., Martínez-Montoya, J. F., Tarango-Arámbula, L. A., Clemente-Sánchez, F., Crosby-Galván, M. M., \& Sánchez-Hermosillo, M. D. (2011). Distribución del ocelote (Leopardus pardalis) en San Luis Potosí, México. Revista Mexicana de Biodiversidad, 82(3), 997-1004.
Martínez-Calderas, J. M., Rosas-Rosas, O. C., Palacio Núñez, J., Martínez-Montoya, J. F., \& Villordo Galván, J. A. (2012). Nuevos registros de tigrillo (Leopardus wiedii) en San Luis Potosí, México. Acta Zoológica Mexicana, 28(2), 482-486.

Massara, R. L., Paschoal, A. M. O., Bailey, L. L., Doherty, P. F., \& Chiarello, A. G. (2016). Ecological interactions between ocelots and sympatric mesocarnivores in protected areas of the Atlantic Forest, southeastern Brazil. Journal of Mammalogy, 97(6), 1634-1644. DOI: $10.1093 /$ jmammal/gyw129

Murray, J. \& Gardner, G. L. (1997). Leopardus pardalis. Mammalian Species, 548, 1-10.

O’ Brien, T. G., Kinnaird, M. F., \& Wibisono, H. T. (2003). Crouching tigers, hidden prey: Sumatran tiger and prey populations in a tropical forest landscape. Animal Conservation, 6(02), 131-139. DOI: 10.1017/ S1367943003003172

Payan, E., Elzirik, E., De Oliveira, T., Leite-Pilman, R., Kelly, M., \& Valderrama, C. (2008). Leopardus wiedii. The IUCN Red List of Threatened Species. Version 2014.2. Retrieved from www.iucnredlist.org

Pérez-Irineo, G., \& Santos-Moreno, A. (2014). Density, distribution, and activity of the ocelot Leopardus pardalis (Carnivora: Felidae) in Southeast Mexican rainforests. Revista Biología Tropical, 62(4), 1421-1432.

Pérez-Irineo, G., \& Santos-Moreno, A. (2016). Abundance and activity patterns of medium-sized felids (Felidae, Carnivora) in Southeastern Mexico. The Southwestern Naturalist, 61(1), 33-39. DOI: 10.1894/0038-4909-61.1.33

Pianka, E. R. (1973). The structure of lizard communities. Annual Review of Ecology and Systematics, 4, 53-74.

Porfirio, G., Foster, V. C., Fonseca, C., \& Sarmento, P. (2016). Activity patterns of ocelots and their potential prey in the Brazilian Pantanal. Mammalian Biology Zeitschrift für Säugetierkunde, 81(5), 511-517. DOI: 10.1016/j.mambio.2016.06.006

Roemer, G. W., Gompper, M. E., \& Van Valkenburgh, B. (2009). The Ecological Role of the Mammalian Mesocarnivore. BioScience, 59(2), 165-173. DOI: 10.1525/bio.2009.59.2.9

Schoener, T. W. (1974). Resource partitioning in ecological communities. Science, 185(4145), 27-39.

Semarnat (Secretaría de Medio Ambiente y Recursos Naturales). (2010). Norma Oficial Mexicana NOM-059-SEMARNAT2010, Protección ambiental-Especies nativas de México de flora y fauna silvestres-Categorías de riesgo y especificaciones para su inclusión, exclusión o cambio-Lista de especies es riesgo. Diario Oficial de la Federación. 30 de diciembre de 2010, Segunda Sección. México. 
Spiegel, M. R. (1991). Estadística. (2a Ed.). Distrito Federal, México: Mc Graw Hill.

Steinberg, M., Taylor, M., \& Kinney, K. (2014). The El Cielo Biosphere Reserve: Forest Cover Changes and Conservation Attitudes in an Important Neotropical Region. The Professional Geographer, 66(3), 403411. DOI: $10.1080 / 00330124.2013 .799994$

Sunquist, M. \& Sunquist, F. (2002). Wild cats of the world. Chicago and London USA: University of Chicago Press.

Thompson, J. N. (1988). Variation in interspecific interactions. Annual Review of Ecology and Systematics, 19, 65-87.

Torres-Romero, E. J., Espinoza-Medinilla, E., LazcanoBarreto, M. A., \& Maffeí, L. (2017). Ecology and conservation of ocelot (Leopardus pardalis) in Northern Quintana Roo, Mexico. Therya, 8(1), 11-18.

Trolle, M. (2003). Mammal survey in the southeastern Pantanal, Brazil. Biodiversity \& Conservation, 12(4), 823-836. DOI: 10.1023/A:1022489426920
Vanderhoff, E. N., Hodge, A. M., Arbogast, B. S., Nilsson, J., \& Knowles, T. W. (2011). Abundance and activity patterns of the margay (Leopardus wiedii) at a midelevation site in the eastern Andes of Ecuador. Mastozoología Neotropical, 18(2), 271-279.

Vargas-Contreras, J. A. \& Hernández-Huerta A. (2001). Distribución altitudinal de la mastofauna en la Reserva de la Biosfera "El Cielo", Tamaulipas, México. Acta Zoológica Mexicana, 82, 83-109.

Winemiller, K. O., \& Pianka, E. R. (1990). Organization in Natural Assemblages of Desert Lizards and Tropical Fishes. Ecological Monographs, 60(1), 27-55. DOI: $10.2307 / 1943025$

Witczuk, J., Pagacz, S., Gliwicz, J., \& Mills, L. S. (2015). Niche overlap between sympatric coyotes and bobcats in highland zones of Olympic Mountains, Washington. Journal of Zoology, 297(3), 176-183. DOI: $10.1111 /$ jzo. 12270

Zar, J. H. (2010). Biostatistical Analysis. Massachusetts, USA: Pearson Prentice-Hall. 